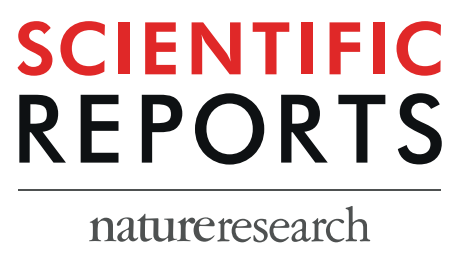

\title{
OPEN Ablation of Myeloid Cell MRP8 Ameliorates Nephrotoxic Serum- induced Glomerulonephritis by Affecting Macrophage Characterization through Intraglomerular Crosstalk
}

\author{
Yusuke Hata ${ }^{1,6}$, Takashige Kuwabara ${ }^{1,2,6 *}$, Kiyoshi Mori ${ }^{2,3,4}$, Youngna Kan ${ }^{2}$, Yuki Sato ${ }^{2}$, \\ Shuro Umemoto ${ }^{1}$, Daisuke Fujimoto ${ }^{1}$, Tomoko Kanki ${ }^{1}$, Yoshihiko Nishiguchi ${ }^{1}$, Hideki Yokoi ${ }^{2}$, \\ Yutaka Kakizoe $^{1}$, Yuichiro Izumi ${ }^{1}$, Motoko Yanagita ${ }^{2,5}$ \& Masashi Mukoyama ${ }^{1,2}$
}

Toll-like receptor 4 (TLR4) and one of its endogenous ligands myeloid-related protein 8 (MRP8 or S100A8), especially expressed in macrophages, play an important role in diabetic nephropathy and autoimmune disorders. However, detailed mechanisms and consequence of MRP8 expression remain unknown, partly due to embryonic lethality of MRP8 knockout mice. In this study, Myeloid lineage cell-specific MRP8 knockout mice were generated, and nephrotoxic serum-induced glomerulonephritis was developed. Mice with conditional ablation of MRP8 gene in myeloid cells exhibited less severe histological damage, proteinuria and inflammatory changes compared to control mice. Mechanism of MRP8 upregulation was investigated using cultured cells. Co-culture of macrophages with mesangial cells or mesangial cell-conditioned media, but not with proximal tubules, markedly upregulated MRP8 gene expression and inflammatory M1 phenotype in macrophages, which was attenuated in MRP8-deleted bone marrow-derived macrophages. Effects of MRP8 deletion was further studied in the context of macrophage-inducible C-type lectin (Mincle), which is critically involved in maintenance of M1 phenotype of macrophages. MRP8 ablation in myeloid cells suppressed the induction of Mincle expression on macrophages in glomerulonephritis. Thus, we propose that intraglomerular crosstalk between mesangial cells and macrophages plays a role in inflammatory changes in glomerulonephritis, and MRP8-dependent Mincle expression in macrophage may be involved in the process.

The prevalence of chronic kidney disease requiring renal replacement therapy is increasing sharply all over the world ${ }^{1}$. Glomerulonephritis is among the second most prevalent causes of end-stage renal disease. Especially, crescentic glomerulonephritis is a still unresolved disease, in which renal prognosis is rapidly declining unless managed properly using glucocorticoids or immunosuppressants ${ }^{2}$. Such situation prompted us to explore the novel therapeutic strategies for crescentic glomerular diseases.

The immune system has originally emerged as a defense mechanism against non-self antigens. In these days, accumulating evidence has shown that the system is also involved in the pathogenesis of various non-communicable diseases, including diabetes, obesity, cancer, and autoimmune disease even without

\footnotetext{
${ }^{1}$ Present address: Department of Nephrology, Kumamoto University Graduate School of Medical Sciences, Kumamoto, Japan. ${ }^{2}$ Department of Nephrology, Kyoto University Graduate School of Medicine, Kyoto, Japan. ${ }^{3}$ Present address: Department of Nephrology and Kidney Research, Shizuoka General Hospital, Shizuoka, Japan. ${ }^{4}$ Present address: School of Pharmaceutical Sciences, University of Shizuoka, Shizuoka, Japan. ${ }^{5}$ Institute for the Advanced Study of Human Biology (ASHBi), Kyoto University, Kyoto, Japan. ${ }^{6}$ These authors contributed equally: Yusuke Hata and Takashige Kuwabara. *email: ktakasea@kumamoto-u.ac.jp
} 
infection ${ }^{3,4}$. Such sterile inflammation is referred to as "chronic inflammation" caused by interactions between pattern recognition receptors such as toll-like receptors and their endogenous ligands, collectively called damage-associated molecular patterns (DAMPs) ${ }^{5}$. Although the precise role for chronic inflammation has yet to be clarified in kidney disease, we and others recently revealed the pathogenic role of toll-like receptor 4 (TLR4) in the development and progression of diabetic nephropathy ${ }^{6-8}$ and crescentic glomerulonephritis ${ }^{9,10}$. With regard to DAMPs, Myeloid-related protein 8 (MRP8, also known as S100A8 or calgranulin A) was originally identified as a cytoplasmic calcium-binding protein in neutrophils and monocytes ${ }^{11}$. Several key reports have shown that MRP8, in association with its family member MRP14 (or S100A9), could act as a potent endogenous ligand for TLR4 in various diseases including septic shock, vascular injury, and autoimmune disorders ${ }^{12-14}$. Besides, we reported that MRP8 expressed in macrophages $(\mathrm{M} \phi)$ could be involved in the progression of diabetic nephropathy in mice and humans ${ }^{6,15,16}$. During these experiments, we unexpectedly observed that glomerular-infiltrated M $\phi$ expressed MRP8 much more robustly than tubulointerstitial M $\phi$, which finding was also observed in human diabetic kidney and glomerulonephritis ${ }^{16,17}$. These observations suggested that the intraglomerular crosstalk may trigger and maintain high MRP8 expression in glomerular-infiltrated $\mathrm{M} \phi$. However, the mechanisms and roles for MRP8 upregulation in glomeruli remain elusive.

Because conventional systemic MRP8 deletion resulted in embryonic lethality ${ }^{18}$, MRP14 knockout mice were alternatively used in most of previous studies, where expression of MRP8 protein was at an undetectable level in myeloid cells ${ }^{19}$. MRP14 deletion in mice, however, does not recapitulate MRP8 deletion completely, as evidenced by no embryonic lethality in those mice. In the present study, we generated myeloid lineage cell-specific MRP8 knockout mice, and nephrotoxic serum-induced glomerulonephritis (NTN) was developed. We found that the ablation of MRP8 in myeloid cells exhibited less severe histological damage, proteinuria and inflammatory changes compared to control mice, and resulted in less M1 phenotype of M $\phi$ interacted with mesangial cells. Besides, the induction of macrophage-inducible C-type lectin (Mincle), which is critically involved in maintenance of M1 phenotype of macrophages ${ }^{20}$, was attenuated in monocytes-M $\phi$ and partly in granulocytes in MRP8-deleted NTN mice. The findings of this study suggested the role of MRP8 in myeloid cells might play important roles in the progression of crescentic glomerulonephritis by affecting $\mathrm{M} \phi$ characterization.

\title{
Results
}

Effective recombination with reduction of MRP8 was achieved in myeloid cell-specific MRP8KO mice. We generated myeloid cell-specific MRP8-deficient (MyM8KO) mice, after confirming successful recombination (Supplemental Fig. S1B). We first evaluated the recombination efficiency in DNA, mRNA and protein levels (Supplemental Fig. S2). As previously reported ${ }^{21}$, lysozyme M-controlled Cre (LysM-Cre) recombinase mRNA was most abundantly expressed in bone-marrow cells, followed by leukocyte-rich organs such as the lung and spleen, but was almost absent in the liver, kidney or heart (Supplemental Fig. S2A). As for genomic DNA recombination, bone marrow and leukocyte-enriched organs, spleen and lung, showed a high efficiency as predicted, but the kidney and liver showed a low efficiency (Supplemental Fig. S2B,C). In the heart, unexpectedly, the targeted allele was also highly deleted by LysM-Cre recombinase. This result could be consistent with a previous report, which described that LysM-GFP was strongly and transiently expressed in embryonic cardiomyocytes ${ }^{22}$. In the mRNA level, LysM-Cre-mediated recombination led to 60-80\% reduction of MRP8 in the bone marrow, lung and spleen. The kidney and liver also showed the reduced MRP8 level, which probably reflected a reduction of MRP8 in the infiltrated and/or residential myeloid-derived cells as in the lung and spleen (Supplemental Fig. S2D). Such reduction tendency was also confirmed in the protein level by Western blotting, which importantly indicated that myeloid lineage cell-specific MRP8 deletion resulted in markedly reduced serum MRP8 levels (Supplemental Fig. S2E).

\begin{abstract}
Upregulation and accumulation of MRP8 in the glomeruli of NTN were effectively attenuated in MyM8KO mice. To investigate the role of myeloid lineage cell-derived MRP8 in glomerulonephritis, we induced NTN in MyM8KO mice (Fig. 1A). After induction of NTN, MRP8 and its binding partner MRP14 were robustly upregulated especially in the isolated glomeruli rather than in whole kidney samples in Cre-negative control mice. Myeloid lineage cell-specific MRP8 deletion effectively suppressed MRP8 but not MRP14 (Fig. 1B). Such reduction was also observed in serum and the kidney tissue by Western blot analysis (Fig. 1C). In NTN mice, MRP8-positive signals were accumulated in glomerular-infiltrated Mac2-positive cells and crescentic lesions (Supplemental Fig. S3). MRP8 deletion significantly abolished these signals in the glomeruli (Fig. 1D,E). On the other hand, some tubular epithelial MRP8 signals remained elevated in MyM8KO mice (Fig. 1D). Faint staining of MRP8 that was broadly distributed in the tubules, possibly reflecting the reabsorption of MRP8 in the proximal tubules, was also decreased in MyM8KO mice. This finding might be compatible with reduced serum MRP8 concentrations in MyM8KO mice (Fig. 1C and Supplemental Fig. S2E).
\end{abstract}

LysM-Cre-mediated recombination was effectively performed in MRP8-positive cells in NTN mice. Next, we developed myeloid lineage cell-specific ZsGreen reporter mice by crossing floxed-STOP ZsGreen transgenic mice and LysM-Cre mice, and induced NTN in the reporter mice to examine the location of LysM-Cre-mediated recombination. As shown in Fig. 2, almost all glomerular MRP8 signals were included in ZsGreen signals, suggesting that LysM-Cre-mediated recombination was effectively performed in MRP8-positive cells in this model.

Ablation of MRP8 in myeloid lineage cells ameliorated glomerulonephritis. We next investigated the effects of myeloid lineage cell-specific MRP8 deletion upon renal injuries. MyM8KO mice revealed effective reduction in proteinuria, glomerular expression of TNF $\alpha$ and IL-1 $\beta$, and the phosphorylation of I $\mathrm{B}$ and NFkB p65 subunit after the induction of NTN (Fig. 3A-C). On the other hand, pro-fibrotic gene expressions, 
A

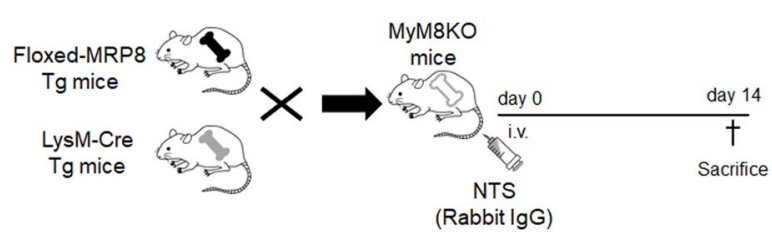

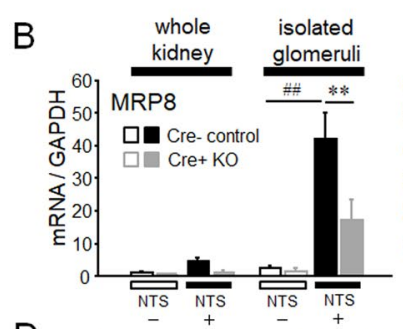

D
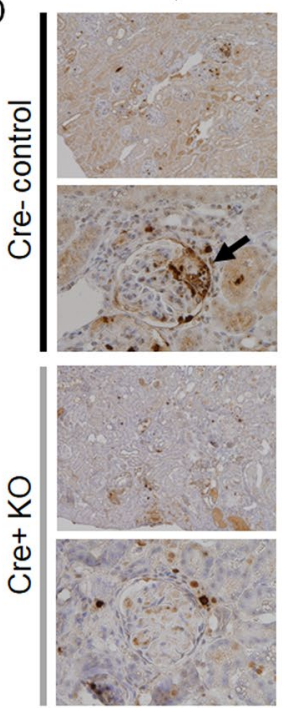
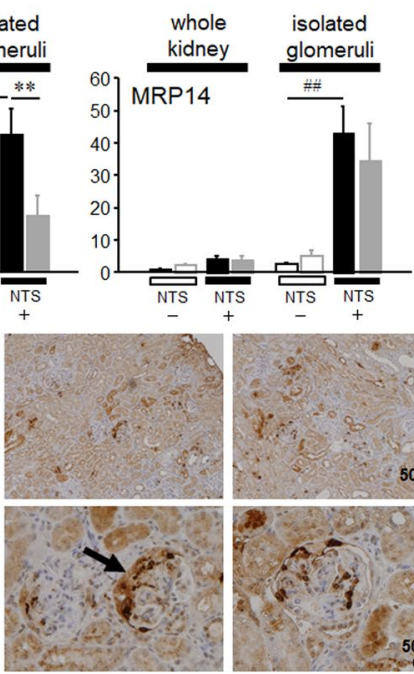

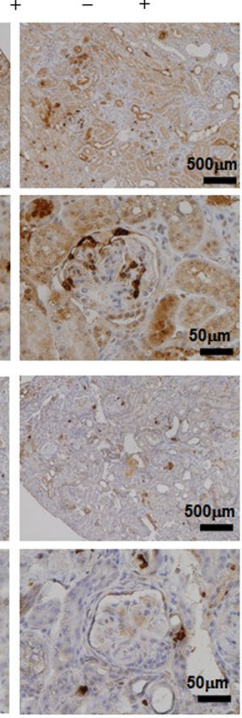

C

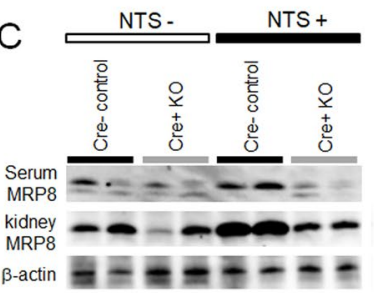

$\mathrm{E}$

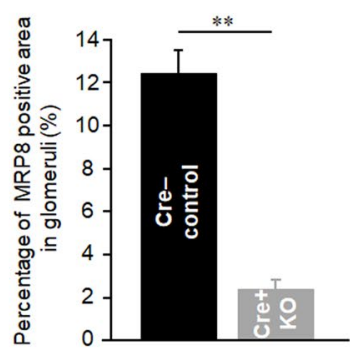

Figure 1. Induction of the experimental nephrotoxic glomerulonephritis (NTN) model in MyM8KO mice and MRP8 expression in this model. (A) To generate MyM8KO mice, floxed-MRP8 transgenic (Tg) mice was crossed with LysM-Cre Tg mice. (B) The mRNA expression of MRP8 and MRP14 was determined by TaqMan real-time RT PCR in the whole kidney and isolated glomeruli. Data are means \pm SEM. $\mathrm{n}=4-6, * * P<0.01$ for Cre- control vs. Cre + KO. ${ }^{\#} P<0.01$ for NTS- vs. NTS + . (C) The amount of MRP8 protein in the serum and kidney was evaluated by Western blotting. (D) Immunohistochemistry of MRP8 in Cre- control and Cre $+\mathrm{KO}$ NTN mice and (E) its quantification analysis of MRP8 positive area in glomeruli. Arrows indicate a crescentic lesion. Original magnification, $\mathrm{x} 4, \mathrm{x} 40$. Data are means \pm SEM. $\mathrm{n}=4-6, * * P<0.01$ for Cre- control vs. Cre + KO. NTS, nephrotoxic serum; MRP8, myeloid-related protein 8; MRP14, myeloid-related protein 14.

including TGF $\beta$, CTGF and fibronectin, were almost comparable between Cre-negative control and MyM8KO mice (Fig. 3B). Deletion of MRP8 did not affect the expression of its receptor TLR4, which was upregulated markedly in the glomeruli of NTN mice (Fig. 3B). Importantly, glomerular exudative lesions evaluated by Masson's trichrome staining were significantly diminished in MyM8KO mice (Fig. 3D).

MRP8 expression in M $\phi$ was induced by co-culture with mesangial cells in a TLR4-independent manner. Because the MRP8 positivity in M $\phi$ was apparently higher in glomerular-infiltrated $M \phi$ compared to tubulointerstitial M $\phi$ in NTN mice (Figs. 1B,D and 2), we performed co-culture experiments to investigate the potential intraglomerular cell-cell crosstalk affecting MRP8 and TLR4 expressions in M $\phi$. The TLR4 mRNA expression in mouse $\mathrm{M} \phi(\mathrm{mM} \phi)$ was not affected by co-culture with either rat mesangial cells (rMes) or proximal tubular cells (rPT), and its expression levels appeared to just reflect the proportion of mM $\phi$ (Fig. 4, left). Similarly, the MRP8 mRNA expression in $\mathrm{mM} \phi$ was not changed by co-culture with $\mathrm{rPT}$; when co-cultured with rMes, however, the MRP8 gene expression in $\mathrm{mM} \phi$ was induced (Fig. 4, right). Such induction was recapitulated by stimulation with rMes-cultured supernatant (Supplemental Fig. S4). Because we and others reported that MRP8 could be induced in M $\phi$ in a TLR4-dependent manner ${ }^{12,16}$, we pharmacologically examined whether the induction of MRP8, and possibly of other pro-inflammatory cytokines, by mesangial cells in vitro was also TLR4-dependent by using E5564, a TLR4 antagonist. The upregulation of MRP8, IL-1 $\beta$ and TNF $\alpha$ by the rMes-cultured medium was not or minimally affected by the treatment with E5564 (Supplemental Fig. S5), suggesting that the induction of MRP8 and other cytokines induced by humoral factors from mesangial cells 


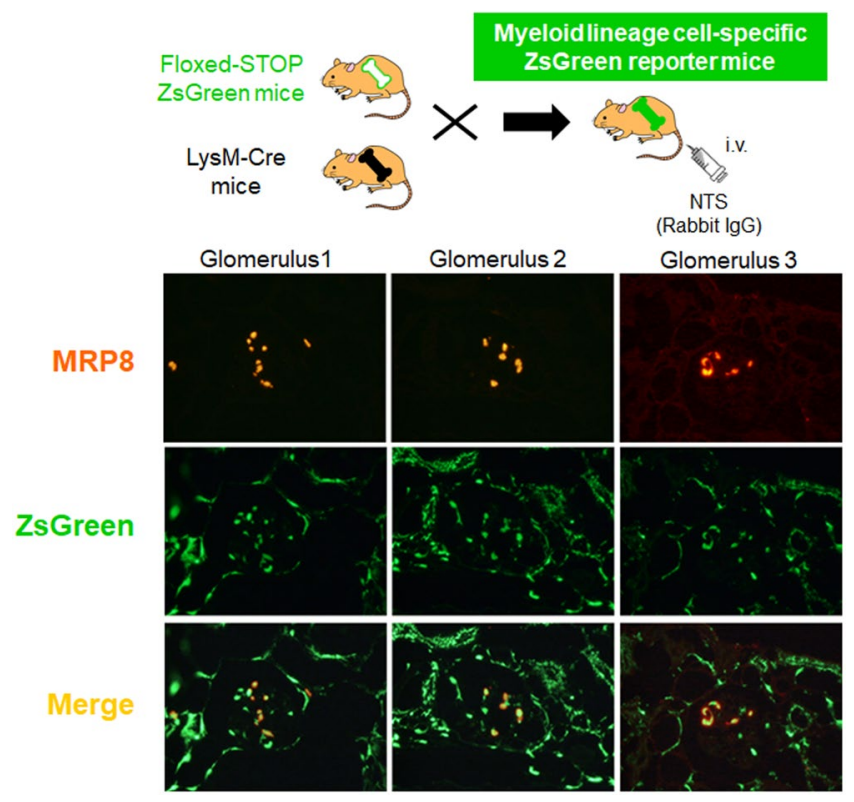

Figure 2. LysM-Cre-mediated recombination was visualized using myeloid lineage cell-specific ZsGreen reporter mice. Original magnification, x 40. LysM, Lysozyme M; NTS, nephrotoxic serum; MRP8, myeloidrelated protein 8.

could be TLR4-independent. Similarly, there was no effect of E5564 on cytokine expression in rPT-cultured medium-treated $\mathrm{M} \phi$ (Supplemental Fig. S6).

Myeloid lineage cell-specific deletion of MRP8 revealed less M1 dominancy in M $\phi . \quad$ After induction of NTN, a pan-M $\phi$ marker F4/80 was markedly increased in isolated glomeruli but was minimally affected by MRP8 deletion in $\mathrm{M} \phi$, whereas the M1/M2 ratio defined by CD11c/Mrc1 was significantly suppressed in MyM8KO mice (Fig. 5A). Then, in order to examine the effects of MRP8 deletion upon M $\phi$ characterization, we generated bone marrow-derived $\mathrm{M} \phi(\mathrm{BMDM})$ from Cre-negative control (Control BMDM) and from MyM8KO mice (KO BMDM), and stimulated them with rMes-cultured medium. The treatment resulted in the increased M1/M2 ratio in Control BMDM; however, KO BMDM showed less M1 dominancy upon stimulation (Fig. 5B). Importantly, more robust stimulation by LPS with skewing toward M1 dominancy in Control BMDM showed an apparently less response in KO BMDM (Supplemental Fig. S7). In addition, we examined mRNA expression levels of interleukin-10 (IL-10) as another M $\phi$ M2 marker, CD4/CD8 ratio as a surrogate marker of T lymphocyte balance, and the receptor for advanced glycation end products (RAGE) as another pattern recognition receptor for MRP8. MyM8KO mice showed a significant suppression of IL-10 mRNA upregulation in the glomeruli after induction of NTN, while there was no difference in the CD4/CD8 ratio or RAGE expression between the genotypes (Supplemental Fig. S8).

Deletion of MRP8 resulted in less stress fiber formation in M $\phi$. Next, we evaluated stress fiber formation in $\mathrm{M} \phi$ by phalloidin staining. Stress fiber-forming cells were defined as cells stretched over $100 \mu \mathrm{m}$ (Supplemental Fig. S9). The rPT-cultured medium did not affect the stress fiber formation both in Control BMDM and KO BMDM (Fig. 6A). On the other hand, rMes-cultured medium significantly increased the occurrence of stress fiber-forming cells in Control BMDM, and this effect was effectively abrogated by MRP8 deletion in KO BMDM (Fig. 6B).

Mincle expression on monocytes- M $\phi$ was attenuated by MRP8 ablation in NTN mice. To examine whether MRP8 deletion in myeloid lineage cells affects the M $\phi$ cell surface markers involved in adhesion and/or migration, FCM analyses were performed for leukocytes in MyM8KO mice (Fig. 7A and Supplemental Fig. S10A). In non-NTN healthy mice, there were no apparent differences in the expression of the markers examined between the genotypes (Supplemental Fig. S10B-E). It is noteworthy that the induction of Mincle expression was effectively attenuated in monocytes-M $\phi$, and partly in granulocytes, from MyM8KO NTN mice (Fig. 7E). In contrast to Mincle, no difference was observed in the expression of L-selectin, another C-type lectin (Fig. 7D).

\section{Discussion}

In the present study, we demonstrated the crucial role of MRP8 expressed in myeloid cells for the development and progression of glomerulonephritis in mice. So far, accumulating evidence has revealed the importance of endogenous molecules released from the cells upon damages or dangers, i.e., DAMPs, in the pathophysiology of a variety of diseases ${ }^{3-5}$. Among them, the role of a heterodimer MRP8/MRP14 has been shown in multiple situations including autoimmune disease $\mathrm{e}^{14}$, cancer $^{23}$, atherosclerotic disease $\mathrm{e}^{13,24,25}$, and kidney injury $\mathrm{y}^{26}$. However, all of these studies were performed using the mice deficient of MRP14, a binding partner of MRP8. To our knowledge, 
A

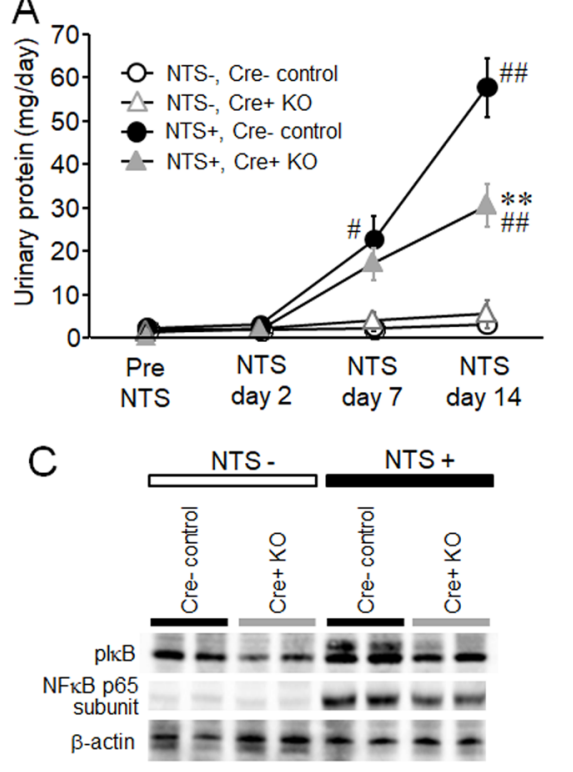

D

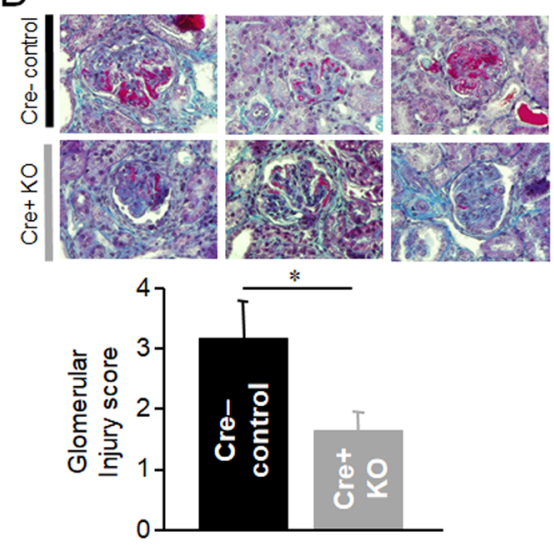

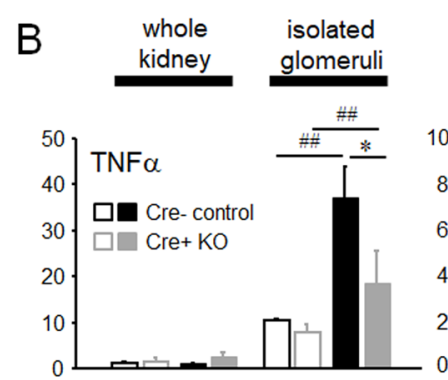
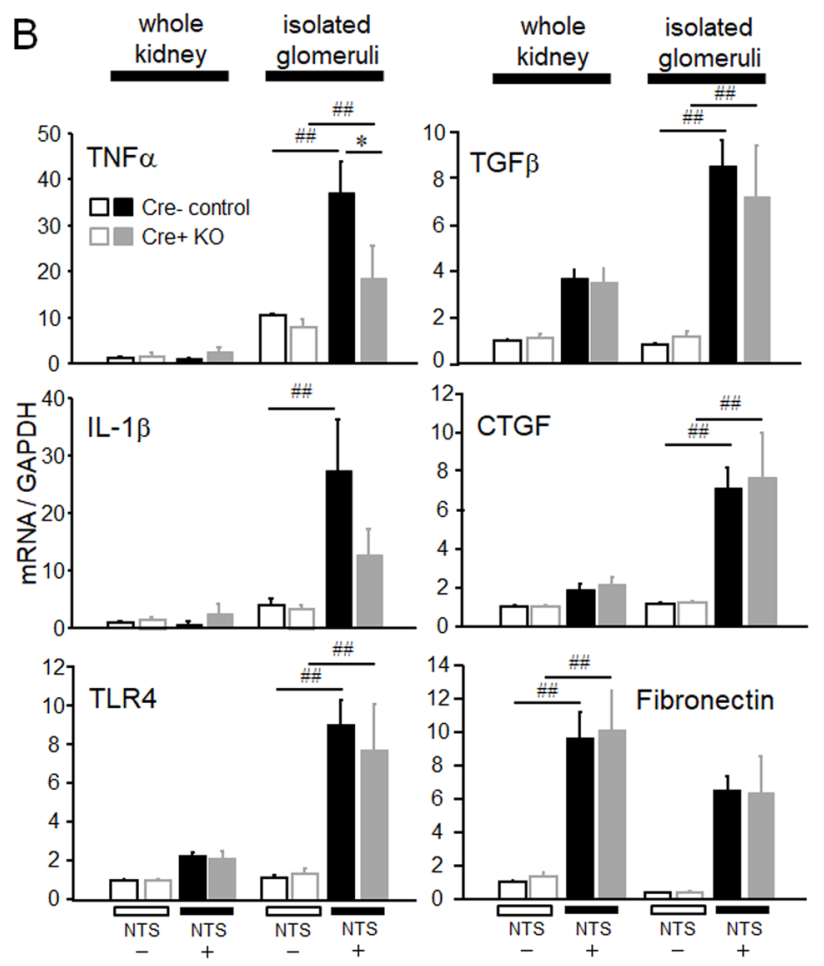

Figure 3. Effects of myeloid lineage cell-specific deletion of MRP8 on renal injuries in NTN mice. (A) Time course of urinary protein after induction of NTN. (B) The mRNA expressions of pro-inflammatory and profibrotic genes and TLR4 determined by TaqMan real-time RT-PCR in the whole kidney and isolated glomeruli.

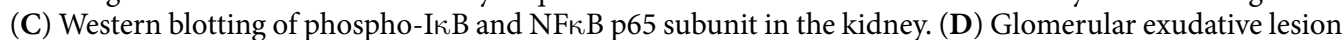
evaluated by Masson's Trichrome staining and its quantified glomerular injury score. Original magnification, $\mathrm{x}$ 40. Data are means \pm SEM. $\mathrm{n}=4-6,{ }^{* *} P<0.01,{ }^{*} P<0.01$ for Cre- control vs. Cre + KO. ${ }^{*} P<0.05,{ }^{* \#} P<0.01$ for NTS- vs. NTS + . NTS, nephrotoxic serum; TNF $\alpha$, tumor necrosis factor-alpha; IL-1 $\beta$, interleukin-1beta; TLR4, toll-like receptor 4; TGF $\beta$, transforming growth factor-beta; CTGF, connective tissue growth factor.

the present study will provide the first direct evidence that loss of MRP8 in myeloid cells could significantly lessen the organ damage in vivo. Furthermore, our data revealed that MRP8 deletion affected the characterization of glomerular-infiltrated cells and peripheral blood $\mathrm{M} \phi$ upon glomerulonephritis. Importantly, enhanced Mincle expression was significantly alleviated in M $\phi$ and partly in neutrophils by the absence of MRP8.

In our experiments, $M \phi M 1$ phenotype was attenuated in parallel with the suppression of gene expression of pro-inflammatory cytokines and the NFKB signaling. In contrast, the expression of Mrcl and Arginase1 (data not shown), the M2 markers, was maintained, similar to that of pro-fibrotic genes such as TGF $\beta$, CTGF or fibronectin (Fig. 3). In this regard, it is important to note that $\mathrm{M} 2 \mathrm{M} \phi$ or fibrocytes could be involved in the development and maintenance of kidney fibrosis ${ }^{26-28}$. Nevertheless, it is also important to note that the early and proper intervention of inflammation with immunosuppressants during an acute phase is critical for the treatment of crescentic glomerulonephritis such as anti-GBM or ANCA-associated glomerulonephritis. In the present study, MyM8KO mice showed the suppression of M1 markers and pro-inflammatory cytokines, along with unaltered, but not aggravated, M2 markers and pro-fibrotic genes after induction of NTN. On the other hand, MyM8KO mice showed significant suppression of IL-10 mRNA expression, which induces the M2 M $\phi$ differentiation, in the glomeruli of NTN (Supplemental Fig. S8). To note, however, there is a heterogeneity of M $\phi$ population involved in kidney injury, being not simply divided into M1 and M2 phenotype. So, we have to consider the diversity including an alternatively activated subset of $\mathrm{M} 2 \mathrm{M} \phi$ rather than just $\mathrm{M} 2$ dominancy ${ }^{29,30}$, and certain changes of 


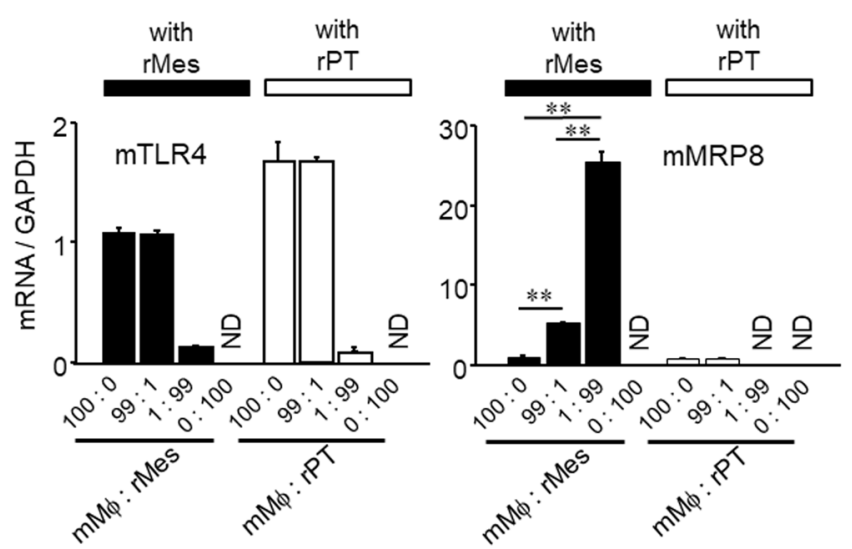

Figure 4. Effects of co-culture with renal intrinsic cells on TLR4 and MRP8 gene expressions in mouse M $\phi$. Mouse TLR4 and MRP8 mRNA expression levels were determined by TaqMan real-time RT-PCR using mousespecific probes. Data are means \pm SEM. $\mathrm{n}=4-8, * * P<0.01 . \mathrm{mM} \phi$, RAW264.7 mouse macrophages; rMes, rat mesangial cells; rPT, rat proximal tubular cells; ND, not detected; mTLR4, mouse toll-like receptor 4; mMRP8, mouse myeloid-related protein 8.

A

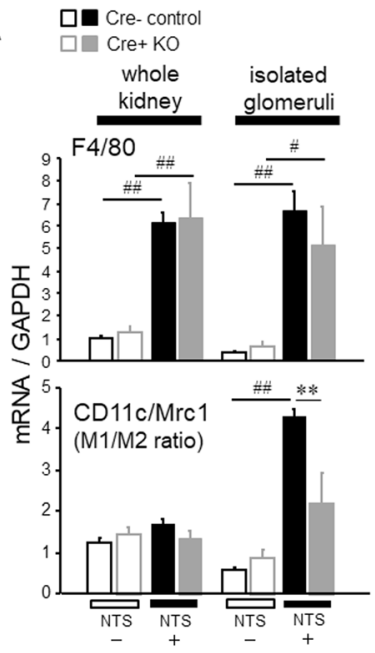

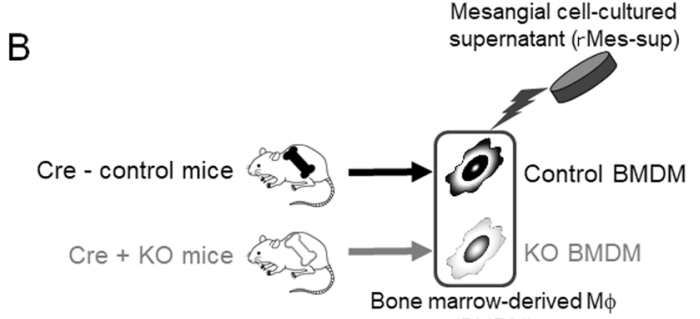

(BMDM)

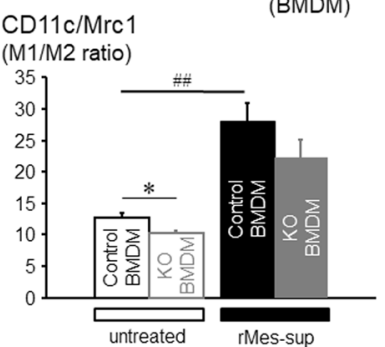

Figure 5. Effects of myeloid lineage cell-specific MRP8 deletion on M $\phi$ polarization in vivo and in vitro. (A) In the kidney of NTN mice, F4/80 mRNA levels and the M1/M2 ratio defined by the CD11c/Mrc1 ratio were evaluated. $\mathrm{n}=4-6,{ }^{* *} P<0.01$ for Cre- control vs. Cre + KO. ${ }^{\#} P<0.05,{ }^{\#} P<0.01$ for NTS- vs. NTS + . (B) $\mathrm{BMDM}$, which were generated from Cre-negative control and from Cre-positive $\mathrm{KO}$ mice, were stimulated with rMes-sup for 24 hours. The M1/M2 ratio was evaluated. $\mathrm{n}=4, * P<0.05$ for Control BMDM vs. KO BMDM.

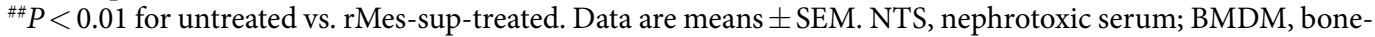
marrow derived macrophages; Mrc1, mannose receptor C type 1.

$\mathrm{M} \phi$ characterization by MRP8 deletion could possibly suppress pro-inflammatory reaction without influence on pro-fibrotic changes in the acute phase of NTN.

We and others reported that MRP8 was upregulated in leukocytes in the diabetic milieu ${ }^{6,31}$, the upregulation of which could be induced by glucolipotoxicity in a TLR4-dependent manner and may represent a positive autocrine loop ${ }^{6,15}$. In the present study, however, the induction of MRP8 in M $\phi$ by mesangial cells appeared to occur in a TLR4-independent manner. MRP8 could also reportedly interact with RAGE ${ }^{23,32}$. Therefore, we examined RAGE expression in the kidney of NTN mice. The expression of RAGE increased dominantly in the isolated glomeruli, just similar to that of TLR4 (Supplemental Fig. S8). The involvement of RAGE in this amelioration could not be excluded in the glomerular lesion at present. Nevertheless, our findings suggest a possibility that the inhibition of MRP8, rather than blockade of its receptor TLR4, could provide another strategy along with additional effects via the RAGE pathway. Another important issue is its local induction upon kidney disease. In our study, MRP8 was robustly and locally induced in the injured glomeruli. Actually, it is reported that glomerular MRP8-positive cells were accumulated in various glomerular diseases such as ANCA-associated glomerulonephritis $^{16}$, IgA nephritis ${ }^{33}$ or membrano-proliferative glomerulonephritis ${ }^{34}$. Vogl T, et al. reported that MRP8/ 

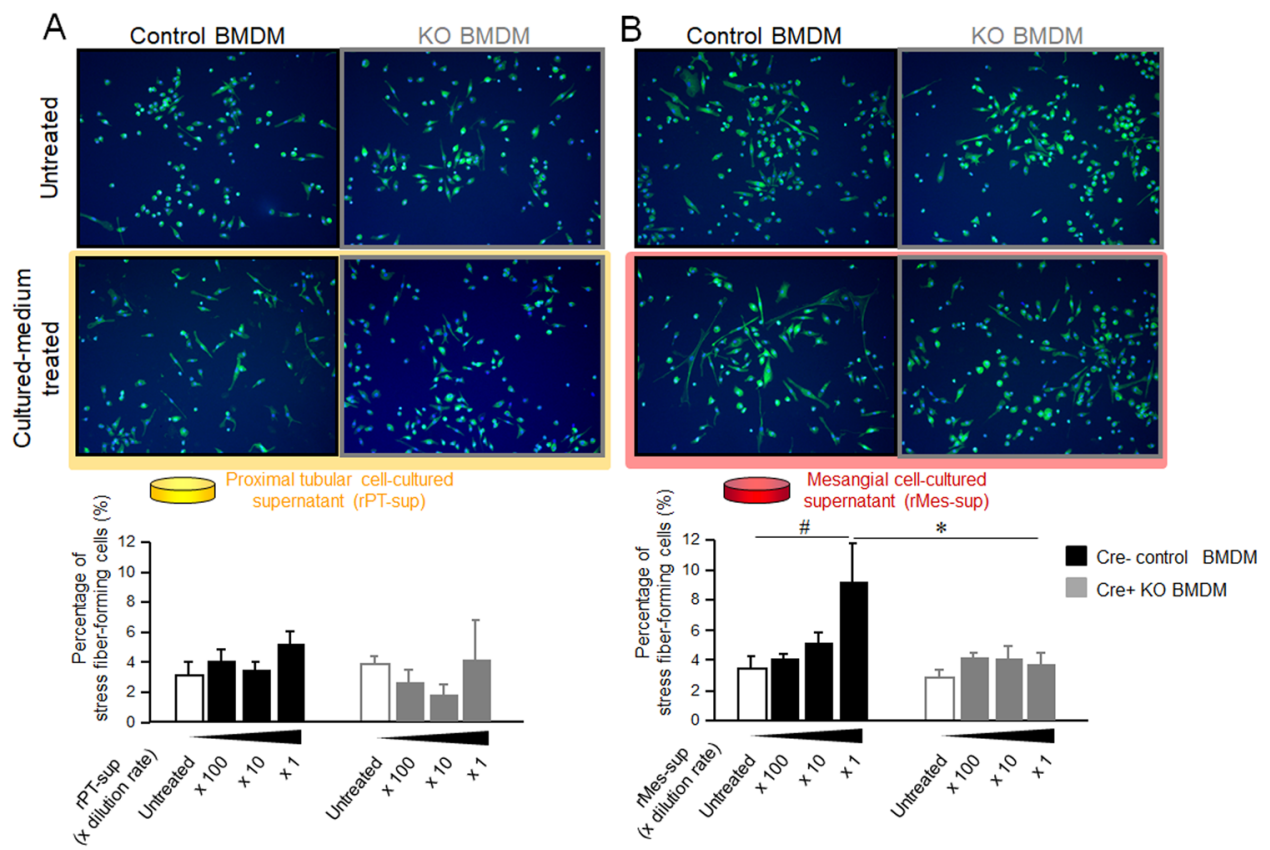

Figure 6. Impact of MRP8 deletion on the stress fiber formation induced by Mes-sup stimulation in BMDM. BMDM, which were generated from Cre-negative control and from Cre-positive KO mice, were incubated with rPT-sup (A) or rMes-sup (B) for 24 hours. Percentage of stress fiber-forming cells was quantified. Data are means \pm SEM. $\mathrm{n}=4-5,{ }^{*} P<0.05$ for Control BMDM vs. KO BMDM. ${ }^{*} P<0.05$ for untreated vs. rMes-suptreated.

MRP14 could indicate the local inflammation in very early phase of the diseases and the prognosis of late disease activities in a model of contact dermatitis or collagen-induced arthritis ${ }^{35}$. Hence, the evaluation of local induction of MRP8 in the kidney tissue would become a useful tool in monitoring renal local inflammation.

There is a variety of cells participating in and contributing to the pathogenesis and progression of crescentic glomerulonephritis. $\mathrm{M} \phi$ are typically involved in the subacute to chronic process following the initial endothelial injury with neutrophil and T cell activation, leading to glomerular exudative lesion ${ }^{36,37}$. In the present study, we found no evidence that loss of MRP8 in myeloid lineage cells affects the lymphocyte characteristics detected by FCM and real-time PCR (Fig. 7, Supplemental Figs. S8 and S10). On the other hand, some of surface molecules affecting adhesion and/or migration were altered in $\mathrm{M} \phi$ and also in neutrophils. Especially, we revealed that Mincle could be an important molecule whose upregulation upon NTN was attenuated by MRP8 deletion. Mincle is a member of the C-type lectin receptor family bound to carbohydrates in a calcium-dependent manner. It has been shown that Mincle plays a pivotal role as a pattern recognition receptor, amplifying innate immunity and activating $\mathrm{M} \phi$ and neutrophils by sensing adjuvant glycolipids, some pathogens and DAMPs released from dying cells $^{38,39}$. It is also recently reported that Mincle is involved in the maintaining M1 phenotype in kidney injury ${ }^{20}$. Although MRP8 induces neutrophil chemotaxis and adhesion by activating L-selectin, another C-type lectin ${ }^{40}$, we could not find any effect on L-selectin by MRP8 deletion. The present study suggested the role of MRP8 in affecting Mincle expression on $\mathrm{M} \phi$ and also weakly on neutrophils. Meanwhile, focusing on the glomerular intrinsic cells, we demonstrated a novel role of mesangial cells in glomerular injury through enhancing MRP8 expression and the following characterization changes in $\mathrm{M} \phi$. We previously reported that mesangial cells could be important in $\mathrm{M} \phi$ infiltration during glomerulonephritis in a CTGF-dependent manner ${ }^{41}$. Therefore, we focused on the intraglomerular crosstalk between mesangial cells and $M \phi$, which induces the expression of MRP8 and M1 dominancy which results in aggravation of kidney injury. Of course, the other glomerular intrinsic cells, especially glomerular endothelial cells, should be noted to interact with $\mathrm{M} \phi$ in progression of glomerulonephritis ${ }^{42}$.

It should be considered carefully that a lysozyme $\mathrm{M}$ promoter is not restricted to $\mathrm{M} \phi$ but can work in other myeloid lineage cells ${ }^{43}$. MRP14 deletion, resulting in loss of circulating MRP8, is shown to differentially modify phenotypic states of neutrophils, $\mathrm{M} \phi$, and dendritic cells in atherosclerosis or adipose tissue inflammation ${ }^{44}$. Hence, we could not deny the possibility that MRP8 in neutrophils contributes to the pathophysiology in NTN. In fact, MRP8-targeting strategies are proposed in various pathological conditions related to the neutrophil activation $25,32,44,45$.

In summary, the present study revealed that MRP8 deletion in myeloid lineage cells resulted in ameliorated glomerular lesions and less M1 phenotype with reduced Mincle expression in a mouse model of crescentic glomerulonephritis. Our data indicate that MRP8 in myeloid cells could potentially aggravate glomerular injury through intraglomerular cell-cell crosstalk affecting M $\phi$ characterization, suggesting the suppression of MRP8 as a potential therapeutic target to ameliorate crescentic glomerulonephritis. 

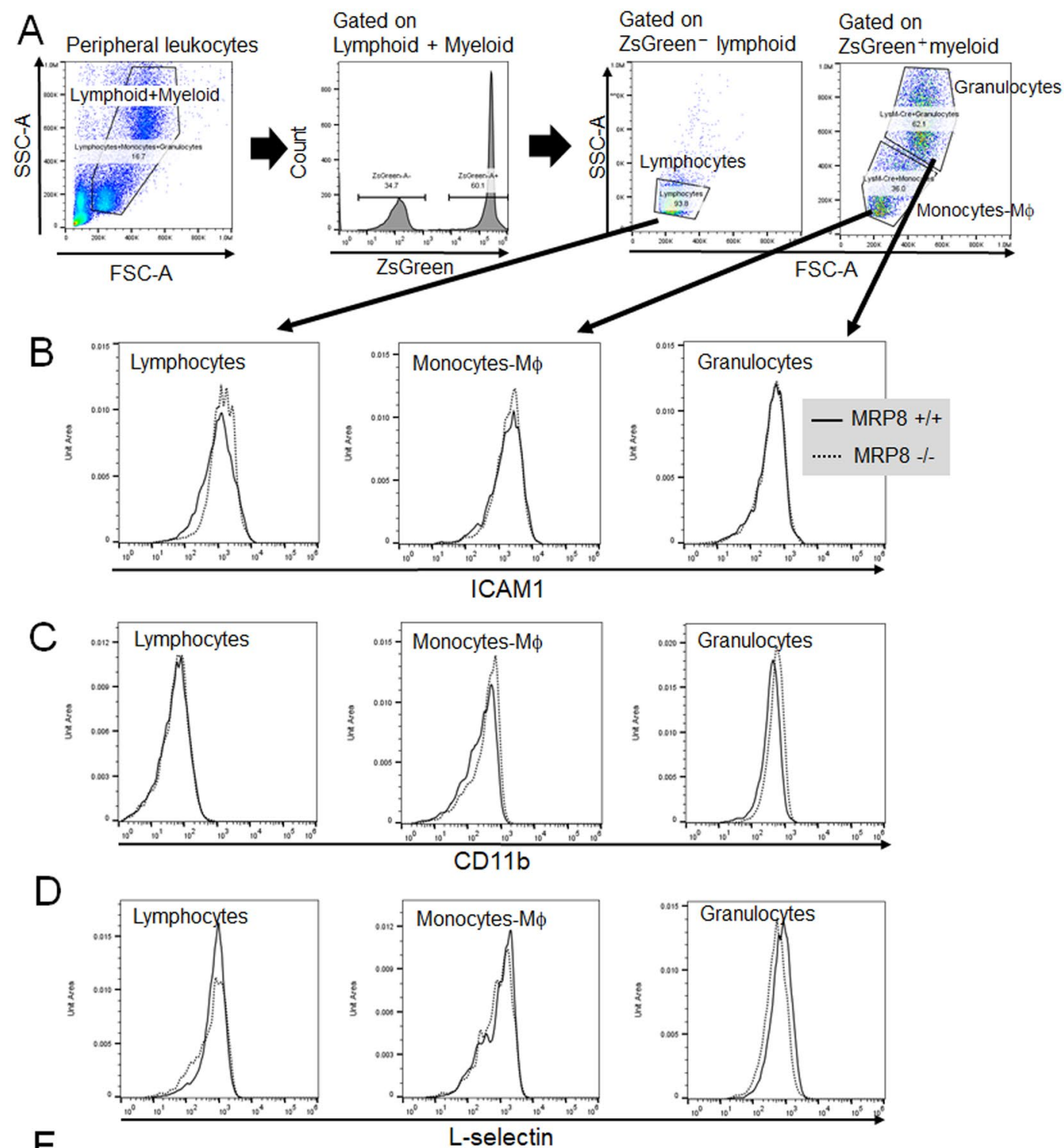

$E$
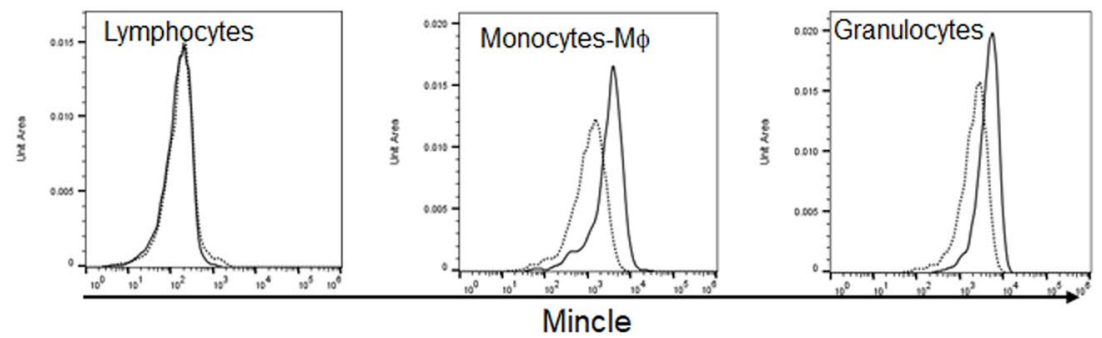

Figure 7. Flow cytometry (FCM) of peripheral blood cells in MyM8KO NTN mice. (A) Sorting strategy for lymphocytes, monocytes and granulocytes in blood. Peripheral leukocytes were separated into lymphoid and myeloid by ZsGreen. Monocytes-M $\phi$ and granulocytes were gated by the conventional method in FSC-SSC plot. (B-E) FCM of ICAM1, CD11b, L-selectin and Mincle in peripheral leukocytes. Solid and dotted lines show the results in flox-negative control and myeloid lineage cell-specific MRP8 deleted mice, respectively.

\section{Methods}

Experimental animals. To generate tissue-specific MRP8 knockout mice, we employed the conventional Cre-loxP system. To flank exons 2 and 3 of the mouse S100a8 gene, which encode a whole coding sequence of MRP8 by loxP sites (MRP8 ${ }^{f l f l}$ ), we used homologous recombination in embryonic stem cells (for more details, see Supplemental information Supplemental Fig. S1). We developed myeloid lineage cell-specific MRP8 knockout mice (MyM8KO) by crossing MRP8fl/fl and lysozyme M-Cre (LysM-Cre) mice, which were purchased from the Jackson Laboratory (Bar Harbor, ME, USA). B6.Cg-Gt(ROSA)26Sor < tm6(CAG-ZsGreen1) 
Hze $>/$ J mice (Jackson Laboratory) were also crossed with LysM-Cre mice to develop myeloid lineage cell-specific ZsGreen reporter mice. Recombination efficacy of MyM8KO mice was evaluated by DNA, mRNA and protein levels with PCR, RT-PCR and Western blotting, respectively. Sequences of PCR primers were 5'-TTCTAGCAGTGTCTAGCAGAAGAGG-3' (forward) and 5'-GAGACCATGTATTTGAGAGGCAGTT-3' (reverse). All animal experiments were approved by Animal Research Committee of Kumamoto University (Certification No. G26-120) along with guidelines. Besides, all experiments were performed in accordance with relevant guidelines and regulations.

Induction of the experimental nephrotoxic glomerulonephritis. Experimental NTN was induced by the conventional method as we previously reported ${ }^{46}$. Briefly, glomeruli were isolated by differential sieving from the ddY mouse renal cortex and disrupted by sonication. The glomerular basement membrane (GBM) was collected by centrifugation, emulsified with complete Freund's adjuvant (CFA; Difco, Detroit, MI, USA) and immunized in rabbits. Mice were immunized by an intraperitoneal injection of $1.0 \mathrm{mg}$ per $20 \mathrm{~g}$ body weight of normal rabbit IgG (ICN, Aurora, OH, USA) emulsified with CFA. Five days later, $0.3 \mathrm{ml}$ per animal of anti-GBM antiserum (nephrotoxic serum [NTS]) or isovolume of control normal rabbit serum was injected from the tail vein. Urine samples were collected with metabolic cages for 24 hours, and urinary protein was measured by the pyrogallol red-molybdate protein dye-binding method (SRL, Tokyo, Japan). Mice were sacrificed, and all tissues were collected at day 14 after induction of glomerulonephritis.

Real-time quantitative RT-PCR. Total RNA was extracted with TRIzol reagent (Invitrogen, Carlsbad, CA, USA) and cDNA in each sample was synthesized by High Capacity cDNA Reverse Transcription Kit (Applied Biosystems, Foster City, CA, USA) from mouse kidneys and glomeruli that were isolated by graded sieving method ${ }^{6,46}$. TaqMan real-time PCR was performed using Premix Ex Taq (Takara Bio, Otsu, Japan) and StepOnePlus Real Time PCR System (Applied Biosystems) (see Supplemental information Supplemental Table S1 for primer and probe sequences). Expression levels of all genes were normalized by Gapdh (internal control) levels which were detected with TaqMan Rodent GAPDH Control Reagents (Applied Biosystems). The mean expression level in the whole kidney of Cre-negative, non-NTS-treated control mice was arbitrarily defined as 1.0.

Histological analysis. Masson's Trichrome staining and immunohistochemistry of MRP8 (requiring antigen retrieval by citrate buffer) and Mac-2 (or Lgals3) ${ }^{6}$ were carried out using kidney sections (thickness 4 $\mu \mathrm{m}$ ) fixed with $4 \%$ buffered paraformaldehyde. Nuclei were counterstained with hematoxylin. All primary antibodies used in this study are shown in Supplemental information Supplemental Table S2. For double staining, the primary antibody for MRP8 was visualized with a DyLight-conjugated secondary antibody (Jackson ImmunoResearch, PA, USA). Immunofluorescence of MRP8 evaluating colocalization with ZsGreen signals was performed with snap frozen cryostat sections $(4 \mu \mathrm{m})$, prefixed with $4 \%$ buffered paraformaldehyde, and with the primary antibody. Photos were taken by a fluorescence microscope (IX81-PAFM; Olympus, Tokyo, Japan). Glomerular exudative lesion, which is defined by the red-colored lesion in Masson's Trichrome staining with extracapillary inflammatory-cells accumulation, of more than 10 glomeruli were measured quantitatively to obtain an average for each mouse using MetaMorph 7.5 software (Molecular Devices, Downingtown, PA, USA). Actually, there were various degrees of exudative lesion in each glomerulus. Therefore, a glomerular injury score was used to assess the degree of glomerular damage as follows: score $0,0 \%$ glomerular exudative area ( $\%$ of glomerulus); $1,0-1 \%$; 2 : $1-3 \%$; 3, 3-6\%; 4, 6-10\%; 5, 10-20\%; 6, over $20 \%$.

Western blot analysis. Proteins extracted from kidney samples were separated by SDS-PAGE, transferred onto PVDF membranes, incubated with primary antibodies and detected with peroxidase-conjugated secondary antibodies and chemiluminescence ${ }^{6,47}$. Gapdh or $\beta$-actin was used as an internal control. All primary antibodies used in this study are shown in Supplemental information Supplemental Table S2.

Flow cytometry (FCM) for leukocytes. For effective sorting of MRP8-targeted cells, MyM8KO mice were crossed with B6.Cg-Gt(ROSA)26Sor < tm6(CAG-ZsGreen1)Hze >/J mice (MyM8KO-ZsG). MyM8KO-ZsG mice enabled us to detect LysM-Cre-induced MRP8-deleted cells with ZsGreen1.

Peripheral blood was collected, and red blood cells were lysed by FCM lysing solution (Santa Cruz Biotechnology, Santa Cruz, CA, USA). White blood cells were collected by centrifugation at $300 \mathrm{x} g$ for $5 \mathrm{~min}-$ utes and resuspended in cold PBS. The cells were incubated with anti-CD16/CD32 for 10 minutes in the dark to block nonspecific binding of antibodies to the Fc $\gamma$ III/II receptors followed by staining with surface markers. Cells were analyzed on the SH800S Cell Sorter (Sony Biotechnology, Tokyo, Japan). Dead cells were excluded by 4',6-diamidino-2-phenylindole (DAPI) labelling. All primary antibodies used in this study are shown in Supplemental information Supplemental Table S2.

Cell culture. Murine M $\phi$ cell line RAW264.7 (mM $\phi)$ and rat renal proximal tubular cell line NRK52E (rPT) were purchased from ATCC (Manassas, VA, USA). Rat mesangial cells (rMes) were kindly provided by Dr. Daisuke Nakano and Prof. Akira Nishiyama (Department of Pharmacology, Kagawa University Medical School, Kagawa, Japan). They were isolated from male Sprague-Dawley rats and were maintained according to previous publications ${ }^{48,49}$. RAW264.7 M $\phi$ were co-cultured with rPT or rMes. Total mRNA was extracted after co-culture for 72 hours, and mRNA expression levels were determined by TaqMan real-time PCR. To detect the gene expression levels of $\mathrm{M} \phi$, TaqMan probes were designed to be mouse-specific complementary sequences. We generated bone marrow-derived M $\phi$ (BMDM) from Cre-negative control and Cre-positive MyM8KO mice to establish the MRP8-deficient M $\phi$. BMDM were generated from mice as described previously ${ }^{50}$. Briefly, following lysis of red blood cells, bone marrow cells were resuspended in medium containing $20 \%$ fetal calf serum and $50 \mathrm{ng} / \mathrm{ml} \mathrm{recom-}$ binant murine GM-CSF (Peprotech, Rocky Hill, NJ, USA), and cultured at $37^{\circ} \mathrm{C}$ in $5 \% \mathrm{CO}_{2}$ atmosphere. On day 
6, medium was replaced with serum-free medium. On day 7, BMDM were used for each experiment. To evaluate the effects of MRP8 deletion in M $\phi$, BMDM were stimulated with rMes-cultured medium or lipopolysaccharide (LPS; Sigma-Aldrich, St Louis, MO, USA). Stress fiber formation in M $\phi$ was evaluated by phalloidin staining. BMDM were fixed with $4 \%$ paraformaldehyde, permeabilized and immunostained with FITC-phalloidin (Sigma Aldrich, St Louis, MO, USA) to visualize actin filaments. Stress fiber-forming cells were defined as cells stretched over $100 \mu \mathrm{m}$. E5564 (Eisai Inc., Woodcliff Lake, NJ, USA) is a synthetic analog of lipid A and a potent and specific antagonist of TLR4, which terminates MD2/TLR4-mediated signaling ${ }^{51}$. To test the effects of E5564 on M $\phi$, RAW264.7 M $\phi$ were stimulated with rMes-cultured supernatant (rMes-sup) or rPT-cultured medium (rPT-sup) for 24 hours. E5564 was added simultaneously with rMes-sup or rPT-sup stimulation. Total RNA was extracted with RNeasy mini kit (Qiagen, Tokyo, Japan).

Statistical analysis. Data are expressed as means \pm SEM. Differences between multiple groups were assessed by two-way factorial ANOVA with Bonferroni's post-hoc test. Comparison between two groups was carried out by unpaired Student's $t$ test. Statistical significance was defined as $p<0.05$.

Received: 27 November 2019; Accepted: 3 February 2020;

Published online: 20 February 2020

\section{References}

1. Liyanage, T. et al. Worldwide access to treatment to end-stage kidney disease: a systematic review. Lancet 385, 1975-1982 (2015).

2. McAdoo, S. P. \& Pusey, C. D. Anti-Glomerular Basement Membrane Disease. Clin. J. Am. Soc. Nephrol. 12, 1162-1172 (2017).

3. Hotamisligil, G. S. Inflammation, metaflammation and immunometabolic disorders. Nat. 542, 177-185 (2017).

4. Donath, M. Y. \& Shoelson, S. E. Type 2 diabetes as an inflammatory disease. Nat. Rev. Immunol. 11, 98-107 (2011).

5. Rosin, D. L. \& Okusa, M. D. Dangers within: DAMP responses to damage and cell death in kidney disease. J. Am. Soc. Nephrol. 22, 416-425 (2011).

6. Kuwabara, T. et al. Exacerbation of diabetic nephropathy by hyperlipidaemia is mediated by Toll-like receptor 4 in mice. Diabetologia 55, 2256-2266 (2012).

7. Lin, M. et al. The TLR4 antagonist CRX-526 protects against advanced diabetic nephropathy. Kidney Int. 83, 887-900 (2013).

8. Lin, M. et al. Toll-like receptor 4 promotes tubular inflammation in diabetic nephropathy. J. Am. Soc. Nephrol. 23, 86-102 (2012).

9. Summers, S. A. et al. Intrinsic renal cell and leukocyte-derived TLR4 aggravate experimental anti-MPO glomerulonephritis. Kidney Int. 78, 1263-1274 (2010).

10. Giorgini, A., Brown, H. J., Sacks, S. H. \& Robson, M. G. Toll-like receptor 4 stimulation triggers crescentic glomerulonephritis by multiple mechanisms including a direct effect on renal cells. Am. J. Pathol. 177, 644-653 (2010).

11. Odink, K. et al. Two calcium-binding proteins in infiltrate macrophages of rheumatoid arthritis. Nat. 330, 80-82 (1987).

12. Vogl, T. et al. Mrp8 and Mrp14 are endogenous activators of Toll-like receptor 4, promoting lethal, endotoxin-induced shock. Nat. Med. 13, 1042-1049 (2007).

13. Croce, K. et al. Myeloid-related protein-8/14 is critical for the biological response to vascular injury. Circulation 120, 427-436 (2009).

14. Loser, K. et al. The Toll-like receptor 4 ligands Mrp8 and Mrp14 are crucial in the development of autoreactive CD8+ T cells. Nat. Med. 16, 713-717 (2010).

15. Kuwabara, T. et al. Macrophage-mediated glucolipotoxicity via myeloid-related protein $8 /$ toll-like receptor 4 signaling in diabetic nephropathy. Clin. Exp. Nephrol. 18, 584-592 (2014).

16. Kuwabara, T. et al. Predictive significance of kidney myeloid-related protein 8 expression in patients with obesity- or type 2 diabetesassociated kidney diseases. PLoS One 9, e88942 (2014).

17. Pepper, R. J. et al. Leukocyte and serum S100A8/S100A9 expression reflects disease activity in ANCA-associated vasculitis and glomerulonephritis. Kidney int. 83, 1150-1158 (2013).

18. Passey, R. J. et al. A null mutation in the inflammation-associated S100 protein S100A8 causes early resorption of the mouse embryo. J. Immunol. 163, 2209-2216 (1999).

19. Hobbs., J. A. et al. Myeloid cell function in MRP-14 (S100A9) null mice. Mol. Cell Biol. 23, 2564-2576 (2003).

20. Lv, L. L. et al. The pattern recognition receptor, Mincle, is essential for maintaining the M1 macrophage phenotype in acute renal inflammation. Kidney Int. 91, 587-602 (2017).

21. Clausen, B. E., Burkhardt, C., Reith, W., Renkawitz, R. \& Forster, I. Conditional gene targeting in macrophages and granulocytes using LysMcre mice. Transgenic Res. 8, 265-277 (1999).

22. Stadtfeld, M., Ye, M. \& Graf, T. Identification of interventricular septum precursor cells in the mouse embryo. Dev. Biol. 302, 195-207 (2007)

23. Hiratsuka, S. et al. The S100A8-serum amyloid A3-TLR4 paracrine cascade establishes a pre-metastatic phase. Nat. Cell Biol. 10, 1349-1355 (2008).

24. Nagareddy, P. R. et al. Hyperglycemia promotes myelopoiesis and impairs the resolution of atherosclerosis. Cell Metab. 17, 695-708 (2013).

25. Nagareddy, P. R. et al. Adipose tissue macrophages promote myelopoiesis and monocytosis in obesity. Cell Metab. 19, 821-835 (2014).

26. Dessing, M. C. et al. The calcium-binding protein complex S100A8/A9 has a crucial role in controlling macrophage-mediated renal repair following ischemia/reperfusion. Kidney Int. 87, 85-94 (2015).

27. Guiteras, R., Flaquer, M. \& Cruzado, J. M. Macrophage in chronic kidney disease. Clin. Kidney J. 9, 765-771 (2016)

28. Wada, T. et al. Involvement of bone-marrow-derived cells in kidney fibrosis. Clin. Exp. Nephrol. 15, 8-13 (2011).

29. Gordon, S. Alternative activation of macrophages. Nat. Rev. Immunol. 3, 23-35 (2003).

30. Ricardo, S. D., van Goor, H. \& Eddy, A. A. Macrophage diversity in renal injury and repair. J. Clin. Invest. 118, 3522-3530 (2008).

31. Jin, Y. et al. The expression of inflammatory genes is upregulated in peripheral blood of patients with type 1 diabetes. Diabetes Care 36, 2794-2802 (2013).

32. Kraakman, M. J. et al. Neutrophil-derived S100 calcium-binding proteins A8/A9 promote reticulated thrombocytosis and atherogenesis in diabetes. J. Clin. Invest. 127, 2133-2147 (2017).

33. Kawasaki, Y. et al. Accumulation of macrophages expressing myeloid-related protein 8 associated with the progression of sclerotic changes in children with IgAnephropathy. Tohoku J. Exp. Med. 218, 49-55 (2009).

34. Kawasaki, Y. et al. Myeloid-related protein 8 expression on macrophages is a useful prognostic marker for renal dysfunction in children with MPGN type 1. Am. J. Kidney Dis. 45, 510-8 (2005).

35. Vogl, T. et al. Alarmin S100A8/S100A9 as a biomarker for molecular imaging of local inflammatory activity. Nat. Commun. 5, 4593 (2014).

36. Jennette, J. C. \& Nachman, P. H. ANCA Glomerulonephritis and Vasculitis. Clin. J. Am. Soc. Nephrol. 12, 1680-1691 (2017) 
37. Furuhashi, K. et al. Serum-starved adipose-derived stromal cells ameliorate crescentic GN by promoting immunoregulatory macrophages. J. Am. Soc. Nephrol. 24, 587-603 (2013).

38. Lu, X., Nagata, M. \& Yamasaki, S. Mincle: 20 years of a versatile sensor of insults. Int. Immunol. 30, 233-239 (2018).

39. Yamasaki, S. et al. Mincle is an ITAM-coupled activating receptor that senses damaged cells. Nat. Immunol. 9, 1179-1188 (2008).

40. Ryckman, C., Vandal, K., Rouleau, P., Talbot, M. \& Tessier, P. A. Proinflammatory activities of S100: proteins S100A8, S100A9, and S100A8/A9 induce neutrophil chemotaxis and adhesion. J. Immunol. 170, 3233-3242 (2003).

41. Toda, N. et al. Crucial role of mesangial cell-derived connective tissue growth factor in a mouse model of anti-glomerular basement membrane glomerulonephritis. Sci. Rep. 7, 42114 (2017).

42. Gabriela, E. G. et al. Inhibition of CXCL16 attenuates inflammatory and progressive phases of anti-glomerular basement membrane antibody-associated glomerulonephritis. Am. J. Pathol. 170, 1485-1496 (2007).

43. Hume, D. A. Applications of myeloid-specific promoters in transgenic mice support in vivo imaging and functional genomics but do not support the concept of distinct macrophage and dendritic cell lineages or roles in immunity. J. Leukoc. Biol. 89, 525-538 (2011).

44. Averill, M. M. et al. S100A9 differentially modifies phenotypic states of neutrophils, macrophages, and dendritic cells: implications for atherosclerosis and adipose tissue inflammation. Circulation 123, 1216-1226 (2011).

45. Pruenster, M. et al. Extracellular MRP8/14 is a regulator of beta2 integrin-dependent neutrophil slow rolling and adhesion. Nat. Commun. 6, 6915 (2015).

46. Suganami, T. et al. Overexpression of brain natriuretic peptide in mice ameliorates immune-mediated renal injury. J. Am. Soc. Nephrol. 12, 2652-2663 (2001).

47. Kuwabara, T. et al. Urinary neutrophil gelatinase-associated lipocalin levels reflect damage to glomeruli, proximal tubules, and distal nephrons. Kidney Int. 75, 285-294 (2009).

48. Suzaki, Y. et al. BMK1 is activated in glomeruli of diabetic rats and in mesangial cells by high glucose conditions. Kidney Int. 65, $1749-1760$ (2004).

49. Nishiyama, A. et al. Involvement of aldosterone and mineralocorticoid receptors in rat mesangial cell proliferation and deformability. Hypertension 45, 710-716 (2005).

50. Fleetwood, A. J., Lawrence, T., Hamilton, J. A. \& Cook, A. D. Granulocyte-macrophage colony-stimulating factor (CSF) and macrophage CSF-dependent macrophage phenotypes display differences in cytokine profiles and transcription factor activities: implications for CSF blockade in inflammation. J. Immunol. 178, 5245-5252 (2007).

51. Barochia, A., Solomon, S., Cui, X., Natanson, C. \& Eichacker, P. Q. Eritoran tetrasodium (E5564) treatment for sepsis: review of preclinical and clinical studies. Expert. Opin. Drug. Metab. Toxicol. 7, 479-494 (2011).

\section{Acknowledgements}

This work was supported in part by research grants from JSPS KAKENHI (Grant Numbers 15K09268, 23390225, 17K09706), the Integration Research for Agriculture and Interdisciplinary Fields from the National Agriculture and Food Research Organization, and Japan Agency for Medical Research and Development (AMED; Grant Number A26, JP18gm5010002 and JP18gm0610011), Daiwa Securities Health Foundation and Japan Society and Technology Agency (Grant Number 2513), Strategic Grant from Center for Metabolic Regulation of Healthy Aging, Kumamoto University Faculty of Life Sciences (Grant Number 009-0909005001011) and Takeda Science Foundation (Grant Number 09700220). We gratefully acknowledge Yuri Ogawa, Hikari Shibuta and Naoko Hirano for technical assistance, and Noriko Nakagawa and Miki Horikiri for secretarial assistance.

\section{Author contributions}

T.Kuwabara and K.M. designed the study. Y.H. and T.Kuwabara performed the experiments and drafted the manuscript. Y.Kan., Y.S. and M.Y advised and supported the techniques of FCM experiments. T.Kuwabara., Y.H., K.M., S.U., D.F., T.Kanki, Y.N., Y. Kakizoe, Y. I., H.Y., M.Y. and M.M. interpreted the results. All authors approved the final version of the manuscript.

\section{Competing interests}

The authors declare no competing interests.

\section{Additional information}

Supplementary information is available for this paper at https://doi.org/10.1038/s41598-020-59970-9.

Correspondence and requests for materials should be addressed to T.K.

Reprints and permissions information is available at www.nature.com/reprints.

Publisher's note Springer Nature remains neutral with regard to jurisdictional claims in published maps and institutional affiliations.

Open Access This article is licensed under a Creative Commons Attribution 4.0 International License, which permits use, sharing, adaptation, distribution and reproduction in any medium or format, as long as you give appropriate credit to the original author(s) and the source, provide a link to the Creative Commons license, and indicate if changes were made. The images or other third party material in this article are included in the article's Creative Commons license, unless indicated otherwise in a credit line to the material. If material is not included in the article's Creative Commons license and your intended use is not permitted by statutory regulation or exceeds the permitted use, you will need to obtain permission directly from the copyright holder. To view a copy of this license, visit http://creativecommons.org/licenses/by/4.0/.

(C) The Author(s) 2020 